\title{
Diurnal Effect of Sub-GeV Dark Matter Boosted by Cosmic Rays
}

\author{
Shao-Feng Ge $\odot^{1,2}$ Jianglai Liu, ${ }^{2,1}$ Qiang Yuan, ${ }^{3,4,5}$ and Ning Zhou $\odot^{2}$ \\ ${ }^{1}$ Tsung-Dao Lee Institute, Shanghai Jiao Tong University, Shanghai 200240, China \\ ${ }^{2}$ School of Physics and Astronomy, Shanghai Jiao Tong University, Key Laboratory for Particle Astrophysics and Cosmology (MOE) \\ and Shanghai Key Laboratory for Particle Physics and Cosmology, Shanghai 200240, China \\ ${ }^{3}$ Key Laboratory of Dark Matter and Space Astronomy, Purple Mountain Observatory, Chinese Academy of Sciences, \\ Nanjing 210033, China \\ ${ }^{4}$ School of Astronomy and Space Science, University of Science and Technology of China, Hefei 230026, China \\ ${ }^{5}$ Center for High Energy Physics, Peking University, Beijing 100871, China
}

(Received 28 May 2020; accepted 15 January 2021; published 5 March 2021)

\begin{abstract}
We point out a new type of diurnal effect for the cosmic ray boosted dark matter (DM). The DM-nucleon interactions not only allow the direct detection of DM with nuclear recoils but also allow cosmic rays to scatter with and boost the nonrelativistic DM to higher energies. If the DM-nuclei scattering cross sections are sufficiently large, the DM flux is attenuated as it propagates through the Earth, leading to a strong diurnal modulation. This diurnal modulation provides another prominent signature for the direct detection of boosted sub-GeV DM, in addition to signals with higher recoil energy.
\end{abstract}

DOI: 10.1103/PhysRevLett.126.091804

Introduction.-Overwhelming evidence from astrophysical and cosmological observations supports the existence of dark matter (DM) [1], which is gravitationally interacting but invisible via electromagnetic interactions. However, the physical nature of DM is poorly understood: the DM identity is unknown with a possible mass spans nearly 80 orders of magnitude [2]. DM direct detection [3] aims to verify the existence of DM particles and measure their interactions via the recoil of target nuclei or electrons, which is believed to be the most direct way to unveil the nature of DM particles $[4,5]$.

Conventionally, direct detection experiments assume the existence of nonrelativistic DM confined in the Galaxy. The gravitational potential of the Galaxy results in an upper limit on the DM velocity of $v_{\chi} \lesssim 600 \mathrm{~km} / \mathrm{s}$ above which DM can escape [6,7]. Because of the energy threshold, which is typically $\mathcal{O}(\mathrm{keV})$, the sensitive mass window of direct detection experiments can only extend down to $\mathcal{O}(1) \mathrm{GeV}$ via the conventional nuclear recoil channel. In recent years, to enhance the sensitivity of detecting sub$\mathrm{GeV} \mathrm{DM}$, many approaches have been explored, including expanding the nuclear recoil detection capability via a low threshold bolometer [8,9] as well as via the Bremsstrahlung [10] and Migdal [11-17] effects, the direct detection of DM-electron recoils [18-23], and various novel detection proposals [24-34].

Published by the American Physical Society under the terms of the Creative Commons Attribution 4.0 International license. Further distribution of this work must maintain attribution to the author(s) and the published article's title, journal citation, and DOI. Funded by SCOAP.
Another interesting possibility has been recently pointed out: nonrelativistic DM can be boosted by cosmic rays (CRs) $[35,36]$ or the solar reflection [37-39]. As long as the DM has finite interactions with matter, it is inevitable for the nonrelativistic DM to be scattered and boosted by the energetic CRs. Although the flux of the CR-boosted DM (CRDM) is a tiny fraction compared to the nonrelativistic DM, it allows explorations of a certain parameter space of sub-GeV DM that was previously inaccessible $[36,40-43]$ in direct detection, thus expanding the sensitive mass region. The CRDM can also produce signals in large neutrino experiments [44-46].

For sub-GeV DM, the DM-nucleon scattering cross section with a contact interaction can be quite sizable, e.g., as large as $10^{-31} \mathrm{~cm}^{2}$ (see [47] and the references in [35]), in contrast to the light mediator case [48]. With this allowed interaction strength, DM particles can experience multiple scatterings and become attenuated when traveling through the Earth [49-52]. If the CRDM flux is anisotropic, a diurnal flux modulation in direct detection experiments is expected $[53,54]$. This is different from the conventional diurnal effect that is mainly for nonrelativistic DM.

Sub-GeV dark matter boosted by cosmic rays.-The spatial and spectral distributions of the CRDM flux depend on the DM and CR distributions in the Galaxy as well as the CRDM scattering processes. Both the DM density and CR intensities vary with their locations in the Galaxy, becoming more concentrated toward the Galactic Center (GC). Therefore, CRs are much more likely to scatter with and boost the DM in the inner Galaxy region. Even for isotropic scattering, the CRDM flux is highly anisotropic over the sky. 
Although the CRDM scattering also affects the CRs, the effect is important only for a very large scattering cross section $\left(\sigma_{\chi p}>10^{-27} \mathrm{~cm}^{2}\right)$ [35]. For simplicity, we assume that the $\mathrm{CR}$ distribution is unaffected. The CRDM emissivity, which describes its spatial and spectrum distributions, is given by [36]

$$
\begin{aligned}
\zeta_{\chi}\left(\boldsymbol{r}, T_{\chi}\right)= & \frac{\rho_{\chi}(|\boldsymbol{r}|)}{m_{\chi}} \sum_{i=p, \mathrm{He}} \int_{T_{i}^{\min }}^{\infty} d T_{i} \frac{n_{\mathrm{CR}, i}\left(\boldsymbol{r}, T_{i}\right)}{T_{\chi}^{\max }\left(T_{i}\right)} \\
& \times v_{i} \sigma_{\chi i} G_{i}^{2}\left(Q^{2}\right),
\end{aligned}
$$

where $T_{i}$ and $T_{\chi}$ are the kinetic energies of the CR species $i$ and the boosted DM with mass $m_{\chi}, T_{i}^{\min }$ is the minimum CR energy required to boost the DM kinetic energy to $T_{\chi}$, and $T_{\chi}^{\max }$ is the maximum DM kinetic energy given $T_{i}$ [36]. There are three main ingredients in Eq. (1): the DM density $\rho_{\chi}(|\boldsymbol{r}|)$ at location $\boldsymbol{r}$, the CR density $n_{\mathrm{CR}, i}$ times its velocity $v_{i}$, and the scattering cross section $\sigma_{\chi i}$. The form factor $G_{i}\left(Q^{2}\right) \equiv 1 /\left(1+Q^{2} / \Lambda_{i}^{2}\right)^{2} \quad$ [55] is a function of the momentum transfer $Q$ with $\Lambda_{p} \approx 770 \mathrm{MeV}$ and $\Lambda_{\mathrm{He}} \approx$ $410 \mathrm{MeV}$ [56] for proton and helium, respectively.

For the DM density $\rho_{\chi}(|\boldsymbol{r}|)$, we adopt the Navarro-FrenkWhite (NFW) [57] profile, $\rho_{\chi}^{\mathrm{nfw}}(r)=\rho_{s} /\left[\left(r / r_{s}\right)(1+\right.$ $\left.\left.r / r_{s}\right)^{2}\right]$ with $r_{s}=20 \mathrm{kpc}$ and $\rho_{s}=0.35 \mathrm{GeV} \mathrm{cm}^{-3}$, as the benchmark DM mass distribution. For comparison, a cored isothermal distribution, $\rho_{\chi}^{\text {iso }}(r)=\rho_{s} /\left[1+\left(r / r_{s}\right)^{2}\right]$ with $r_{s}=5 \mathrm{kpc}$ and $\rho_{s}=1.56 \mathrm{GeV} \mathrm{cm}^{-3}$, is also studied. These parameters correspond to a local DM density of $0.4 \mathrm{GeV} \mathrm{cm}^{-3}$ in our Solar System [58] for both profiles. The difference between the two profiles and more details are given in the Supplemental Material [59]. The amplitudes of the diurnal modulation vary by only around $7 \%$ for different density profiles.

For the CR contribution in Eq. (1), we employ the GALPROP [70] code (version 54) to simulate its distribution. In this Letter, we only consider the dominating proton and helium species of CRs and leave the rest, in particular electrons and positrons, for future discussions. For the detailed CR model parameters and the resulting CR spatial distribution, please see the Supplemental Material [59].

The DM-nucleus interaction is the least known part in Eq. (1). For simplicity, we assume that the DM-nucleus cross section $\sigma_{\chi A}$ has a coherent enhancement,

$$
\sigma_{\chi A}=\sigma_{\chi p} A^{2}\left[\frac{m_{A}\left(m_{\chi}+m_{p}\right)}{m_{p}\left(m_{\chi}+m_{A}\right)}\right]^{2},
$$

where $\sigma_{\chi n}=\sigma_{\chi p}$ is the constant DM-nucleon cross section, while $m_{p}$ and $m_{A}$ are the proton and nuclear masses for the CR. For $m_{\chi} \ll m_{p}, m_{A}$, the enhancement mainly comes from the $A^{2}$ factor. Extra enhancement may come from $\left(m_{\chi}+m_{p}\right)^{2} / m_{p}^{2}$ when $m_{\chi}$ goes beyond $m_{p}$. The dipole hadronic form factor $G_{i}\left(Q^{2}\right)$ in Eq. (1) suppresses the interaction at the large momentum transfer $Q$.
The CRDM flux arriving at the Earth along a given direction $\hat{\boldsymbol{n}}$ is a line-of-sight integral of all contributions along the way,

$$
\frac{d \Phi}{d T_{\chi}}\left(\hat{\boldsymbol{n}}, T_{\chi}\right)=\frac{1}{4 \pi} \int \zeta_{\chi}\left(\boldsymbol{r}, T_{\chi}\right) d l .
$$

Figure 1 shows the relative all-sky maps of the CRDM fluxes in the Galactic coordinate, a spherical coordinate with the Sun as its center, the latitude measuring the angle above or below the galactic plane, and the longitude measuring the azimuth angle from the GC. The peak value at the GC is set to 1. The top (bottom) panel presents the NFW (Isothermal) profile. The CRDM fluxes are clearly anisotropic, with the maximum (the GC direction) and the minimum differing by about 2 orders of magnitude. To match the grid resolution of GALPROP, we set the NFW density within $0.5 \mathrm{kpc}$ of the $\mathrm{GC}$ to $\rho(0.5 \mathrm{kpc})$. This approximation has a negligible effect on the diurnal modulation, as shown in the Supplemental Material [59].

Figure 2 shows the CRDM spectra from the GC direction for different DM masses. The number density $\rho_{\chi} / m_{\chi}$ in Eq. (1) accounts for the decrease of CRDM flux for larger DM masses. On the other hand, on average the maximum boost occurs when $m_{\chi}$ approaches the mass of the incident
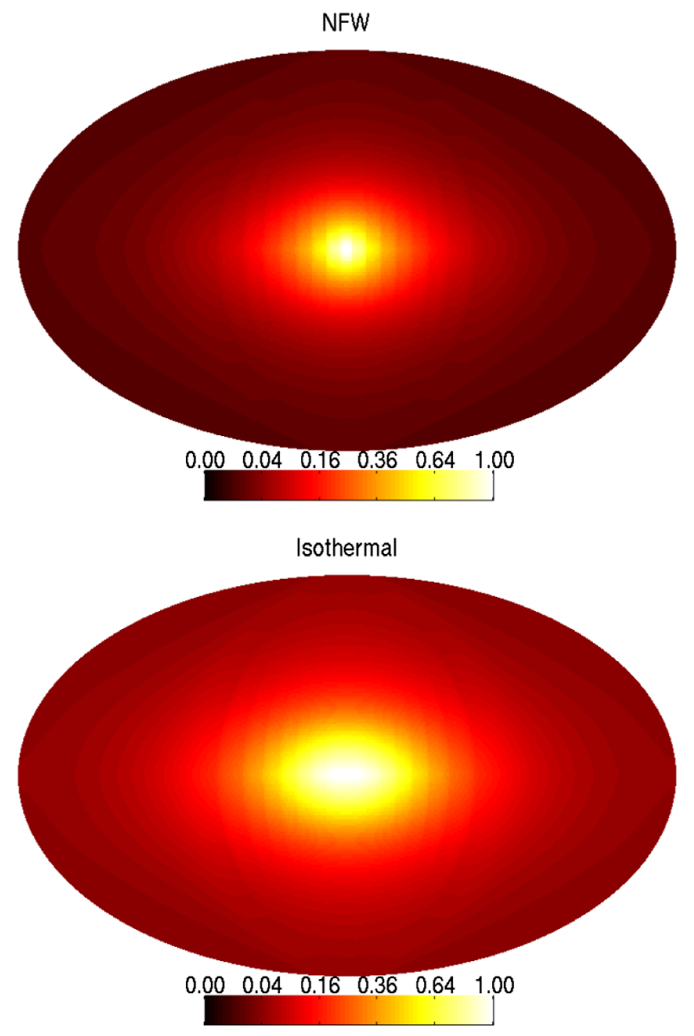

FIG. 1. Relative sky maps of CRDM fluxes in the Galactic coordinates with amplitude in the GC direction set to unity. The upper and lower panels are for the NFW and Isothermal DM density profiles, respectively. 


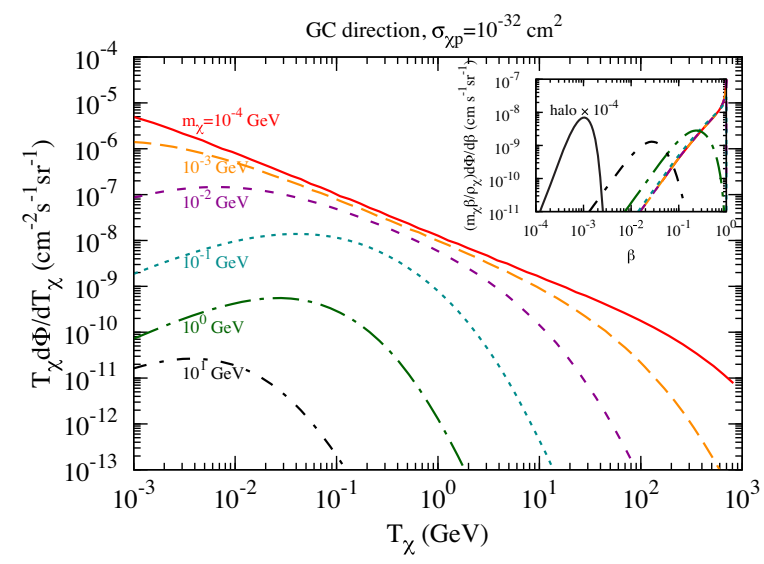

FIG. 2. The CRDM energy spectra at the GC direction for DM masses $10^{-4}, 10^{-3}, 10^{-2}, 0.1,1.0$, and $10 \mathrm{GeV}$ from top to bottom. The scattering cross section $\sigma_{\chi p}$ is assumed to be $10^{-32} \mathrm{~cm}^{2}$. The inset is the distribution of DM velocities, $\beta=v / c$, compared to the Maxwellian distribution of the Standard DM Halo. For a clear comparison, we rescale the Standard DM Halo curve by $10^{-4}$ (labeled as halo $\times 10^{-4}$ in the inset) so that all curves have a similar height.

proton or helium, manifesting in the change of spectrum shape for different energies. At the high energy end, the spectra are suppressed by the form factor $G_{i}\left(Q^{2}\right)$ with $Q^{2}=2 m_{\chi} T_{\chi}$. We also show the nonrelativistic DM velocity distribution predicted by the Standard DM Halo model (labeled as halo $\times 10^{-4}$ ) in Fig. 2 for comparison.

We find that the CRDM spectra depend very weakly on directions, mainly due to the similar CR spectral shapes throughout the Galaxy. For simplicity, in the following discussion we will separate the energy and angular distributions of the CRDM fluxes.

Earth attenuation.-With a large enough scattering cross section, the DM can frequently scatter with matter when traveling through the Earth [19,49-52], transferring its kinetic energy to matter nuclei. Although the decelerated DM particle may still reach the detector, the DM energy spectrum is shifted lower, leading to fewer events above the detector energy threshold. For simplicity, we use the average nucleon numbers, $\bar{A}_{m}=24$ in the Earth mantle and $\bar{A}_{c}=54$ in the Earth core, to approximate the matter compositions [71]. As a concrete example, for $\sigma_{\chi p}=10^{-32} \mathrm{~cm}^{2}$, the mean free path, $L_{\text {free }} \equiv m_{N} /\left(\rho_{N} \sigma_{\chi A}\right)$, is around 2.7 or $17 \mathrm{~km}$ in the Earth core or mantle omitting the form factor effects. Similar attenuation happens in the atmosphere, but due to the 3 orders of magnitude lower density, the effect is only visible at much larger cross sections.

The differential CRDM flux $d \Phi\left(\hat{\boldsymbol{n}}, l, T_{\chi}\right) / d \ln T_{\chi}$, at the distance $l$ through the Earth, is a combination of the loss of DM particles to an energy lower than $T_{\chi}$ and the gain from a higher energy $T_{\chi}^{\prime}$ to $T_{\chi}$. For an incoming DM particle with a higher energy $T_{\chi}^{\prime}$, the nuclear recoil energy $T_{r}$ is evenly distributed in the range $0 \leq T_{r} \leq T_{\chi}^{\prime}\left(T_{\chi}^{\prime}+\right.$ $\left.2 m_{\chi}\right) /\left(T_{\chi}^{\prime}+m_{\mu}\right) \equiv T_{r}^{\max }\left(T_{\chi}^{\prime}\right) \quad$ with reduced mass $m_{\mu} \equiv\left(m_{N}+m_{\chi}\right)^{2} / 2 m_{N}$. Because of energy conservation, $T_{\chi}$ is also evenly distributed: $T_{\chi}^{\prime}\left(m_{\mu}-2 m_{\chi}\right) /\left(T_{\chi}^{\prime}+m_{\mu}\right) \leq$ $T_{\chi} \leq T_{\chi}^{\prime}$. For a given $T_{\chi}$, the DM particles with energy $T_{\chi}^{\prime}$ in the range $T_{\chi} \leq T_{\chi}^{\prime} \leq m_{\mu} T_{\chi} /\left(m_{\mu}-2 m_{\chi}-T_{\chi}\right)$ increases the flux at $T_{\chi}$. The CRDM flux evolution contains two contributions [44]:

$$
\begin{aligned}
\frac{\partial}{\partial l} \frac{d \Phi\left(l, T_{\chi}\right)}{d \ln T_{\chi}}= & \frac{\rho_{N}(l)}{m_{N}} \sigma_{\chi N}\left[-\frac{d \Phi\left(l, T_{\chi}\right)}{d \ln T_{\chi}} w_{\mathrm{FF}}\left(T_{\chi}\right)\right. \\
& \left.+\int \frac{d \Phi\left(l, T_{\chi}^{\prime}\right)}{d \ln T_{\chi}^{\prime}} \frac{T_{\chi}\left(T_{\chi}^{\prime}+m_{\mu}^{N}\right)}{T_{\chi}^{\prime}\left(T_{\chi}^{\prime}+2 m_{\chi}\right)} G_{N}^{2}\left(Q^{2}\right) d \ln T_{\chi}^{\prime}\right] .
\end{aligned}
$$

The weight factor is defined as $w_{\mathrm{FF}} \equiv \int G_{N}^{2}\left(Q^{2}\right) d Q^{2} / Q_{\max }^{2}$, and the factor $T_{\chi} / T_{r}^{\max }$ in the second term comes from the differential cross section $d \sigma=\sigma d T_{r} / T_{r}^{\max }=\sigma d \ln T_{\chi}\left(T_{\chi} / T_{r}^{\max }\right)$. The attenuated DM flux can be obtained by integrating Eq. (4) step by step over the traversed distance. Figure 3 shows the attenuated CRDM fluxes with different nadir angles to the underground detector. To be realistic, we consider a detector $2 \mathrm{~km}$ underground. Then for $\theta_{\text {nadir }}=90^{\circ}$, the DM needs to travel $160 \mathrm{~km}$ before reaching the detector, corresponding to nine mean free paths in the mantle. The CRDM flux at medium energy is largely reduced first and then goes back up at high energy. The limited attenuation at high energy is due to the highly suppressed weight factor $w_{\mathrm{FF}}\left(T_{\chi}\right)$ in Eq. (4). Consequently, the CRDM is much more energetic than the nonrelativistic DM (see the inset of Fig. 3) and can produce recoil events with much higher energy. This makes direct detection experiments sensitive to sub-GeV DMs.

Boosted diurnal effect.-The two anisotropies from the Earth and the Galaxy lead to the diurnal effect. First, the path lengths that DM particles traverse are anisotropic since

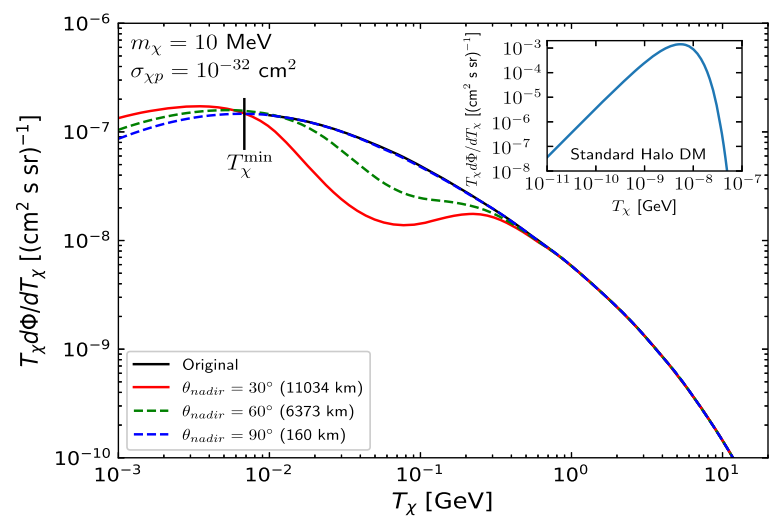

FIG. 3. The attenuated CRDM spectra for the nadir angles $\theta_{\text {nadir }}=30^{\circ} \quad$ (red), $60^{\circ} \quad$ (green), and $90^{\circ}$ (blue) with $\sigma_{\chi p}=10^{-32} \mathrm{~cm}^{2}, m_{\chi}=10 \mathrm{MeV}$, and the detector at a depth of $2 \mathrm{~km}$. For comparison, we also show the original standard halo DM flux distribution in the inset. 


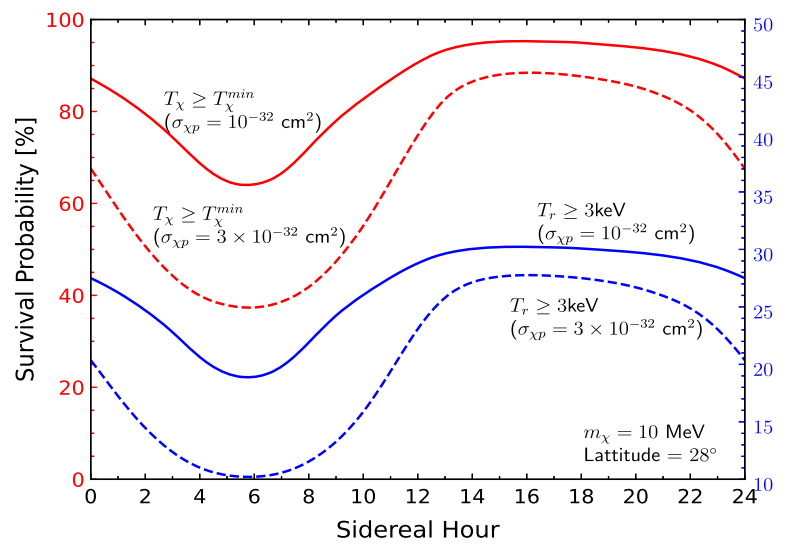

FIG. 4. The survival probability of CRDM arriving at an underground lab at latitude $28^{\circ} \mathrm{N}$ and a depth of $2 \mathrm{~km}$ vs the sidereal hour relative to the number of DM particles arriving at the Earth for two different cross sections $\sigma_{\chi p}=1(3) \times 10^{-32} \mathrm{~cm}^{2}$. The red curves correspond to the total CRDM arriving at the detector with $T_{\chi} \geq T_{\chi}^{\text {min }}$, and the blue curves are those above the detector threshold $\left(T_{r}>3 \mathrm{keV}\right.$ for a liquid xenon detector).

the underground lab is close to the Earth surface and its depth is typically much smaller than the Earth radius. Second, the CRDM flux is strongly peaked toward the GC due to the DM and CR distributions. The CRDM flux is thus significantly attenuated by the Earth when the GC and the detector are on opposite sides of the Earth but much less affected if they are on the same side. To avoid confusion with the usual diurnal effect for nonrelativistic DM $[53,54]$, we call this the "boosted diurnal effect."

Figure 4 shows the diurnal modulation of the CRDM at a direct detection experiment located at a latitude of $28^{\circ} \mathrm{N}$ (approximate location of the China Jinping Underground Laboratory) and a depth of $2 \mathrm{~km}$ underground. Within one sidereal day, the underground lab rotates around the Earth axis and its position is parameterized by the sidereal hour in the range between 0 and 24 hours. We define a survival probability as the ratio between the attenuated CRDM flux in the underground lab and the one arriving the Earth. At a cross section of $1 \times 10^{-32} \mathrm{~cm}^{2}$, we observe significant "boosted diurnal modulation" with the survival probability varying in the range of $64 \%-95 \%$. For comparison, we also show the curves for a cross section of $3 \times 10^{-32} \mathrm{~cm}^{2}$ where a larger modulation can be observed. Given the DM energy $T_{\gamma}$, the nuclear recoil has a wide distribution, $0 \leq T_{r} \leq T_{r}^{\max }\left(T_{\chi}\right)$, and hence only a fraction, $1-T_{t h} / T_{r}^{\max }\left(T_{\chi}\right)$, can pass the detection threshold, leading to a reduction from the red curve to the blue one in Fig. 4.

Instead of via a numerical integration of Eq. (4), the curves in Fig. 4 are obtained by Monte Carlo simulations. Since the spectrum of the CRDM is almost independent of its direction, it is a good approximation to first sample the direction of the incoming DM particles according to the sky map in Fig. 1 and then sample the boosted DM kinetic energy $T_{\chi}$ according to the spectrum in Fig. 2. The incident
DM particle would then experience multiple scatterings when crossing the Earth. For each interaction step, we first sample the distance that the DM particle travels before the next scattering based on the mean free path and then sample the reduced kinetic energy. The simulation stops when the DM particle reaches the underground detector or drops below the detection threshold.

Imposing the detection threshold on the nuclear recoil energy $T_{r} \geq 3 \mathrm{keV}$ for a liquid xenon detector [72] would reduce the event rate but still keep the modulation behavior as illustrated in Fig. 4. This is because the diurnal modulation mainly comes from the high recoil part, as illustrated in Fig. 3. For two years of data at a benchmark liquid xenon detector PandaX-4T (5.6 tons $\times$ year exposure) [73], on average 8.1 (55) events are expected for $\sigma_{\chi p}=1(3) \times 10^{-32} \mathrm{~cm}^{2}$ and $m_{\chi}=10 \mathrm{MeV}$, which is quite significant compared to the background level [74]. For the same detector, the event rate and hence the sensitivity is roughly independent of the DM mass for $m_{\chi} \lesssim 0.1 \mathrm{GeV}$. In addition to a quadratic scaling with the cross section, one from the CRDM production and the other from its detection, the event rate is suppressed once the attenuation from the Earth becomes dominating for a sufficiently large cross section $\left(\sim 10^{-28} \mathrm{~cm}^{2}\right)$ [36]. The cross section region that this technique can probe spans roughly 4 orders of magnitude.

Another factor is the scattering angle, which leads to deflection [19]. For the relativistic CRDM with typical $1 \mathrm{GeV}$ kinetic energy, mass $m_{\chi}=10 \mathrm{MeV}$, and typical momentum transfer $Q \approx \Lambda \approx 200 \mathrm{MeV}$ [56], the scattering angle is $3^{\circ}-5^{\circ}$. Although not completely negligible, the scattering angle does not affect the diurnal modulation effect due to the following arguments. For the peak region of Fig. 4, the DM from the GC only needs to penetrate $\mathcal{O}(1) \mathrm{km}$. With a mean free path of around $17 \mathrm{~km}$, most CRDMs experience only one scattering at most. Therefore, the peak region would not be affected significantly. Multiple scatterings will further suppress the valley region of the curve and therefore enhance the modulation effect.

The recoil energy spectra for incident CRDMs along different nadir angles in a liquid xenon detector are shown in Fig. 5. Since the recoil energy can reach $\mathcal{O}(1 \mathrm{MeV})$, observing a high energy recoil event is a smoking gun for the CRDM, especially when the detector and the GC are on the same side of the Earth. However, these energetic recoils may excite target isotopes and therefore may no longer be simple nuclear recoils. The signal identification strategy for such events needs more experimental study. Statistically, the boosted diurnal modulation can help to identify such high energy recoil signals and suppress the background, which is expected to be constant over time. A more detailed analysis with real data will appear in a future work. 


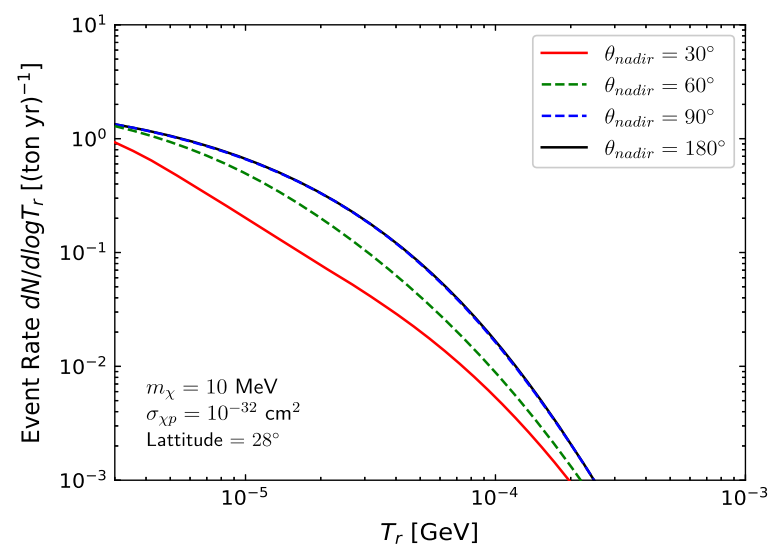

FIG. 5. The nuclear recoil spectrum, including the $3 \mathrm{keV}$ detector threshold, for a xenon detector with 1 ton year exposure. To illustrate the attenuation effect, each curve corresponds to the integrated DM flux at a given nadir angle $\theta_{\text {nadir }}$.

Conclusion.-The CRDM provides a possibility for the conventional DM direct detection experiments to extend their sensitive window to the sub-GeV mass range via the detection of boosted DM events that produce a higher energy recoil above threshold. If the DM-nucleon cross section is sufficiently large, the CRDM is significantly attenuated when traveling through the Earth. Because of the anisotropies of the CRDM flux and the Earth attenuation, the event rate and energy spectrum exhibit a characteristic diurnal modulation, which is a powerful signature to suppress background and enhance sensitivities to sub$\mathrm{GeV}$ DM. Future work can use the electron component in the $\mathrm{CR}$ and extend this exploration to DM-electron interactions. In addition, future directional detection experiments may directly image the anisotropic sky map of the CRDM. The modulation discussed in this Letter may also apply to the boosted DM scenario [75-77].

S. F. G. is sponsored by the Double First Class start-up fund (WF220442604) provided by Tsung-Dao Lee Institute and Shanghai Jiao Tong University, the Shanghai Pujiang Program (20PJ1407800), and the National Natural Science Foundation of China (No. 12090064). Q. Y. is supported by the National Natural Science Foundation of China (Nos. 11722328 and 11851305), the 100 Talents Program of the Chinese Academy of Sciences, and the Program for Innovative Talents and Entrepreneur in Jiangsu. J. L. and N.Z. are supported by the National Natural Science Foundation of China (Nos. 11525522, 11775141, and 12090061), a grant from the Ministry of Science and Technology of China (No. 2016YFA0400301), and a grant from Office of Science and Technology, Shanghai Municipal Government (No. 18JC1410200) and the Hongwen Foundation in Hong Kong. J. L. also acknowledges support from the Tencent Foundation. This work is also supported in part by the Chinese Academy of Sciences Center for Excellence in Particle Physics (CCEPP). The authors are grateful to Dr. Xiang-Yi Cui for checking the calculations.
[1] B. L. Young, A survey of dark matter and related topics in cosmology, Front. Phys. (Beijing) 12, 121201 (2017).

[2] G. B. Gelmini, The hunt for dark matter, arXiv:1502.01320.

[3] M. W. Goodman and E. Witten, Detectability of certain dark matter candidates, Phys. Rev. D 31, 3059 (1985).

[4] J. Liu, X. Chen, and X. Ji, Current status of direct dark matter detection experiments, Nat. Phys. 13, 212 (2017).

[5] M. Schumann, Direct detection of WIMP dark matter: Concepts and status, J. Phys. G 46, 103003 (2019).

[6] N. Bozorgnia, R. Catena, and T. Schwetz, Anisotropic dark matter distribution functions and impact on WIMP direct detection, J. Cosmol. Astropart. Phys. 12 (2013) 050.

[7] J. Zavala and C. S. Frenk, Dark matter haloes and subhaloes, Galaxies 7, 81 (2019).

[8] A. H. Abdelhameed et al. (CRESST Collaboration), First results from the CRESST-III low-mass dark matter program, Phys. Rev. D 100, 102002 (2019).

[9] S. Pirro and P. Mauskopf, Advances in bolometer technology for fundamental physics, Annu. Rev. Nucl. Part. Sci. 67, 161 (2017).

[10] C. Kouvaris and J. Pradler, Probing Sub-GeV Dark Matter with Conventional Detectors, Phys. Rev. Lett. 118, 031803 (2017).

[11] M. Ibe, W. Nakano, Y. Shoji, and K. Suzuki, Migdal effect in dark matter direct detection experiments, J. High Energy Phys. 03 (2018) 194.

[12] D. Baxter, Y. Kahn, and G. Krnjaic, Electron ionization via dark matter-electron scattering and the Migdal effect, Phys. Rev. D 101, 076014 (2020).

[13] R. Essig, J. Pradler, M. Sholapurkar, and T. T. Yu, Relation between the Migdal Effect and Dark Matter-Electron Scattering in Isolated Atoms and Semiconductors, Phys. Rev. Lett. 124, 021801 (2020).

[14] D. S. Akerib et al. (LUX Collaboration), Results of a Search for Sub-GeV Dark Matter Using 2013 LUX Data, Phys. Rev. Lett. 122, 131301 (2019).

[15] E. Armengaud, C. Augier, A. Benoit, A. Benoit, L. Berge et al. (EDELWEISS Collaboration), Searching for low-mass dark matter particles with a massive Ge bolometer operated above-ground, Phys. Rev. D 99, 082003 (2019).

[16] Z. Z. Liu et al. (CDEX Collaboration), Constraints on SpinIndependent Nucleus Scattering with Sub-GeV Weakly Interacting Massive Particle Dark Matter from the CDEX-1B Experiment at the China Jinping Underground Laboratory, Phys. Rev. Lett. 123, 161301 (2019).

[17] E. Aprile et al. (XENON Collaboration), Search for Light Dark Matter Interactions Enhanced by the Migdal Effect or Bremsstrahlung in XENON1T, Phys. Rev. Lett. 123, 241803 (2019).

[18] R. Essig, J. Mardon, and T. Volansky, Direct detection of sub-GeV dark matter, Phys. Rev. D 85, 076007 (2012); Sec. IV-V of M. Battaglieri, et al., US cosmic visions: New ideas in dark matter 2017: Community report, arXiv:1707.04591.

[19] T. Emken, R. Essig, C. Kouvaris, and M. Sholapurkar, Direct detection of strongly interacting sub-GeV dark matter via electron recoils, J. Cosmol. Astropart. Phys. 09 (2019) 070.

[20] R. Essig, A. Manalaysay, J. Mardon, P. Sorensen, and T. Volansky, First Direct Detection Limits on sub-GeV Dark Matter from XENON10, Phys. Rev. Lett. 109, 021301 (2012); R. Essig, T. Volansky, and T. T. Yu, New constraints 
and prospects for sub-GeV dark matter scattering off electrons in Xenon, Phys. Rev. D 96, 043017 (2017); E. Aprile et al. (XENON Collaboration), Light Dark Matter Search with Ionization Signals in XENON1T, Phys. Rev. Lett. 123, 251801 (2019).

[21] P. Agnes et al. (DarkSide Collaboration), Constraints on Sub-GeV Dark-Matter Electron Scattering from the DarkSide-50 Experiment, Phys. Rev. Lett. 121, 111303 (2018).

[22] D. W. Amaral et al. (SuperCDMS Collaboration), Constraints on low-mass, relic dark matter candidates from a surface-operated SuperCDMS single-charge sensitive detector, Phys. Rev. D 102, 091101 (2020).

[23] R. Essig, M. Fernandez-Serra, J. Mardon, A. Soto, T. Volansky, and T. T. Yu, Direct detection of sub-GeV dark matter with semiconductor targets, J. High Energy Phys. 05 (2016) 046; O. Abramoff, L. Barak, I. M. Bloch, L. Chaplinsky, M. Crisler et al. (SENSEI Collaboration), SENSEI: Direct-Detection Constraints on Sub-GeV Dark Matter from a Shallow Underground Run Using a Prototype SkipperCCD, Phys. Rev. Lett. 122, 161801 (2019); A. AguilarArevalo, D. Amidei, D. Baxter, G. Cancelo, B. A. CervantesVergara et al. (DAMIC Collaboration), Constraints on Light Dark Matter Particles Interacting with Electrons from DAMIC at SNOLAB, Phys. Rev. Lett. 123, 181802 (2019).

[24] V. Zacek, Search for dark matter with moderately superheated liquids, Nuovo Cimento A 107, 291 (1994).

[25] Y. Hochberg, Y. Zhao, and K. M. Zurek, Superconducting Detectors for Superlight Dark Matter, Phys. Rev. Lett. 116, 011301 (2016); Y. Hochberg, T. Lin, and K. M. Zurek, Detecting ultralight bosonic dark matter via absorption in superconductors, Phys. Rev. D 94, 015019 (2016).

[26] Y. Hochberg, M. Pyle, Y. Zhao, and K. M. Zurek, Detecting superlight dark matter with Fermi-degenerate materials, J. High Energy Phys. 08 (2016) 057.

[27] K. Schutz and K. M. Zurek, Detectability of Light Dark Matter with Superfluid Helium, Phys. Rev. Lett. 117, 121302 (2016); S. Knapen, T. Lin, and K. M. Zurek, Light dark matter in superfluid helium: Detection with multiexcitation production, Phys. Rev. D 95, 056019 (2017).

[28] S. Derenzo, R. Essig, A. Massari, A. Soto, and T. T. Yu, Direct detection of sub-GeV dark matter with scintillating targets, Phys. Rev. D 96, 016026 (2017).

[29] P. C. Bunting, G. Gratta, T. Melia, and S. Rajendran, Magnetic bubble chambers and sub-GeV dark matter direct detection, Phys. Rev. D 95, 095001 (2017).

[30] Y. Hochberg, Y. Kahn, M. Lisanti, K. M. Zurek, A. G. Grushin, R. Ilan, S. M. Griffin, Z. F. Liu, S. F. Weber, and J. B. Neaton, Detection of sub-MeV dark matter with threedimensional dirac materials, Phys. Rev. D 97, 015004 (2018).

[31] N. A. Kurinsky, T. C. Yu, Y. Hochberg, and B. Cabrera, Diamond detectors for direct detection of sub-GeV dark matter, Phys. Rev. D 99, 123005 (2019).

[32] Y. Hochberg, I. Charaev, S. W. Nam, V. Verma, M. Colangelo, and K. K. Berggren, Detecting Sub-GeV Dark Matter with Superconducting Nanowires, Phys. Rev. Lett. 123, 151802 (2019).

[33] T. Trickle, Z. Zhang, and K. M. Zurek, Direct Detection of Light Dark Matter with Magnons, Phys. Rev. Lett. 124, 201801 (2020); S. Chigusa, T. Moroi, and K. Nakayama,
Detecting light boson dark matter through conversion into magnon, Phys. Rev. Lett. 124, 201801 (2020).

[34] N. Kurinsky, D. Baxter, Y. Kahn, and G. Krnjaic, A dark matter interpretation of excesses in multiple direct detection experiments, Phys. Rev. D 102, 015017 (2020).

[35] C. V. Cappiello, K. C. Y. Ng, and J. F. Beacom, Reverse direct detection: Cosmic ray scattering with light dark matter, Phys. Rev. D 99, 063004 (2019).

[36] T. Bringmann and M. Pospelov, Novel Direct Detection Constraints on Light Dark Matter, Phys. Rev. Lett. 122, 171801 (2019).

[37] C. Kouvaris, Probing light dark matter via evaporation from the sun, Phys. Rev. D 92, 075001 (2015).

[38] H. An, M. Pospelov, J. Pradler, and A. Ritz, Directly Detecting MeV-scale Dark Matter via Solar Reflection, Phys. Rev. Lett. 120, 141801 (2018).

[39] T. Emken, C. Kouvaris, and N. G. Nielsen, The sun as a subGeV dark matter sccelerator, Phys. Rev. D 97, 063007 (2018).

[40] J. Alvey, M. Campos, M. D. Fairbairn, and T. You, Detecting Light Dark Matter via Inelastic Cosmic Ray Collisions, Phys. Rev. Lett. 123, 261802 (2019).

[41] J. B. Dent, B. Dutta, J. L. Newstead, and I. M. Shoemaker, Bounds on cosmic ray-boosted dark matter in simplified models and its corresponding neutrino-floor, Phys. Rev. D 101, 116007 (2020).

[42] W. Wang, L. Wu, J. M. Yang, H. Zhou, and B. Zhu, Sub-GeV gravity-mediated dark matter in direct detections, J. High Energy Phys. 12 (2020) 072.

[43] R. Plestid, V. Takhistov, Y. D. Tsai, T. Bringmann, A. Kusenko, and M. Pospelov, New constraints on millicharged particles from cosmic-ray production, Phys. Rev. D 102, 115032 (2020).

[44] Y. Ema, F. Sala, and R. Sato, Light Dark Matter at Neutrino Experiments, Phys. Rev. Lett. 122, 181802 (2019).

[45] C. V. Cappiello and J. F. Beacom, Strong new limits on light dark matter from neutrino experiments, Phys. Rev. D 100, 103011 (2019).

[46] G. Guo, Y. L. S. Tsai, and M. R. Wu, Probing high-energy light dark matter with IceCube, J. Cosmol. Astropart. Phys. 10 (2020) 049.

[47] G. Krnjaic and S. D. McDermott, Implications of BBN bounds for cosmic ray upscattered dark matter, Phys. Rev. D 101, 123022 (2020).

[48] K. Bondarenko, A. Boyarsky, T. Bringmann, M. Hufnagel, K. Schmidt-Hoberg, and A. Sokolenko, Direct detection and complementary constraints for sub-GeV dark matter, J. High Energy Phys. 03 (2020) 118.

[49] G. D. Starkman, A. Gould, R. Esmailzadeh, and S. Dimopoulos, Opening the window on strongly interacting dark matter, Phys. Rev. D 41, 3594 (1990).

[50] G. D. Mack, J. F. Beacom, and G. Bertone, Towards closing the window on strongly interacting dark matter: Far-reaching constraints from Earth's heat flow, Phys. Rev. D 76, 043523 (2007).

[51] D. Hooper and S. D. McDermott, Robust constraints and novel gamma-ray signatures of dark matter that interacts strongly with nucleons, Phys. Rev. D 97, 115006 (2018).

[52] T. Emken and C. Kouvaris, How blind are underground and surface detectors to strongly interacting dark matter?, Phys. Rev. D 97, 115047 (2018). 
[53] J. Collar and F. Avignone, Diurnal modulation effects in cold dark matter experiments, Phys. Lett. B 275, 181 (1992); J. Collar, F. Avignone, R. Brodzinski, H. Miley, J. Reeves, E. Garcia, A. Morales, J. Morales, R. NunezLagos, C. Saenz, and J. Villar, Bounds on diurnal modulations from the COSME-II dark matter experiment, Nucl. Phys. B, Proc. Suppl. 28A, 297 (1992); D. Di Gregorio, A. Gattone, H. Huck, A. Macchiavelli, S. Gil, J. Collar, and F. Avignone III, Effects of diurnal modulation in direct cold matter searches. The experiment in Sierra Grande, arXiv: astro-ph/9311049; J. Collar and F. Avignone III, Surface sputtering from cold dark matter interactions: Proposed search for its diurnal modulation, Astropart. Phys. 3, 37 (1995); F. Hasenbalg, D. Abriola, J. I. Collar, D. E. Di Gregorio, A. O. Gattone, H. Huck, D. Tomasi, and I. Urteaga, Cold dark matter identification: Diurnal modulation revisited, Phys. Rev. D 55, 7350 (1997); F. S. Ling, P. Sikivie, and S. Wick, Diurnal and annual modulation of cold dark matter signals, Phys. Rev. D 70, 123503 (2004); R. Foot, Diurnal modulation due to self-interacting mirror and hidden sector dark matter, J. Cosmol. Astropart. Phys. 04 (2012) 014; R. Bernabei et al. (DAMA-LIBRA Collaboration), Model independent result on possible diurnal effect in DAMA/LIBRA-phase1, Eur. Phys. J. C 74, 2827 (2014); R. Foot and S. Vagnozzi, Diurnal modulation signal from dissipative hidden sector dark matter, Phys. Lett. B 748, 61 (2015); D. Akerib et al. (LUX Collaboration), Search for annual and diurnal rate modulations in the LUX experiment, Phys. Rev. D 98, 062005 (2018).

[54] J. Vergados and C. Moustakidis, The diurnal variation of the wimp detection event rates in directional experiments, arXiv:0912.3121; A. Bandyopadhyay and D. Majumdar, On diurnal and annual variations of directional detection rates of dark matter, Astrophys. J. 746, 107 (2012); R. J. Creswick, S. Nussinov, and F. T. Avignone, Direction dependence and diurnal modulation in dark matter detectors, Astropart. Phys. 35, 62 (2011); J. D. Vergados and C. C. Moustakidis, WIMP event rates in directional experiments: The diurnal variation signature, Central Eur. J. Phys. 9, 628 (2011).

[55] C. Perdrisat, V. Punjabi, and M. Vanderhaeghen, Nucleon electromagnetic form factors, Prog. Part. Nucl. Phys. 59, 694 (2007).

[56] I. Angeli, A consistent set of nuclear rms charge radii: Properties of the radius surface R(N,Z), At. Data Nucl. Data Tables 87, 185 (2004).

[57] J. F. Navarro, C. S. Frenk, and S. D. M. White, A Universal density profile from hierarchical clustering, Astrophys. J. 490, 493 (1997).

[58] R. Catena and P. Ullio, A novel determination of the local dark matter density, J. Cosmol. Astropart. Phys. 08 (2010) 004.

[59] See Supplemental Material, which includes [60-69], at http://link.aps.org/supplemental/10.1103/PhysRevLett.126 .091804 for the comparison of different DM profiles.

[60] Q. Yuan, C. R. Zhu, X. J. Bi, and D. M. Wei, Secondary cosmic-ray nucleus spectra disfavor particle transport in the Galaxy without reacceleration, J. Cosmol. Astropart. Phys. 11 (2020) 027.

[61] M. Aguilar et al. (AMS Collaboration), Observation of the Identical Rigidity Dependence of $\mathrm{He}, \mathrm{C}$, and $\mathrm{O}$ Cosmic
Rays at High Rigidities by the Alpha Magnetic Spectrometer on the International Space Station, Phys. Rev. Lett. 119, 251101 (2017).

[62] M. Aguilar et al. (AMS Collaboration), Observation of New Properties of Secondary Cosmic Rays Lithium, Beryllium, and Boron by the Alpha Magnetic Spectrometer on the International Space Station, Phys. Rev. Lett. 120, 021101 (2018).

[63] G. L. Case and D. Bhattacharya, A new sigma-d relation and its application to the galactic supernova remnant distribution, Astrophys. J. 504, 761 (1998).

[64] A. Cummings, E. Stone, B. Heikkila, N. Lal, W. Webber, G. J hannesson, I. Moskalenko, E. Orlando, and T. Porter, Galactic cosmic rays in the local interstellar medium: Voyager 1 observations and model results, Astrophys. J. 831, 18 (2016).

[65] M. Aguilar et al. (AMS Collaboration), Precision Measurement of the Proton Flux in Primary Cosmic Rays from Rigidity $1 \mathrm{GV}$ to $1.8 \mathrm{TV}$ with the Alpha Magnetic Spectrometer on the International Space Station, Phys. Rev. Lett. 114, 171103 (2015).

[66] M. Aguilar et al. (AMS Collaboration), Precision Measurement of the Helium Flux in Primary Cosmic Rays of Rigidities $1.9 \mathrm{GV}$ to $3 \mathrm{TV}$ with the Alpha Magnetic Spectrometer on the International Space Station, Phys. Rev. Lett. 115, 211101 (2015).

[67] Y. Yoon et al. (CREAM Collaboration), Proton and helium spectra from the CREAM-III flight, Astrophys. J. 839, 5 (2017).

[68] Q. An et al. (DAMPE Collaboration), Measurement of the cosmic-ray proton spectrum from $40 \mathrm{GeV}$ to $100 \mathrm{TeV}$ with the DAMPE satellite, Sci. Adv. 5, eaax3793 (2019).

[69] L. Gleeson and W. Axford, Solar modulation of galactic cosmic rays, Astrophys. J. 154, 1011 (1968).

[70] A.W. Strong and I. V. Moskalenko, Propagation of cosmic-ray nucleons in the galaxy, Astrophys. J. 509, 212 (1998).

[71] W. F. McDonough and R. Arevalo, Uncertainties in the composition of Earth, its core and silicate sphere, J. Phys. Conf. Ser. 136, 022006 (2008).

[72] X. Cui et al. (PandaX-II Collaboration), Dark Matter Results From 54-Ton-Day Exposure of PandaX-II Experiment, Phys. Rev. Lett. 119, 181302 (2017), supplementary material.

[73] H. Zhang et al. (PandaX Collaboration), Dark matter direct search sensitivity of the PandaX-4T experiment, Sci. Chin. Phys. Mech. Astron. 62, 31011 (2019).

[74] Q. Wang et al. (PandaX-II Collaboration), An improved evaluation of the neutron background in the PandaX-II experiment, Sci. Chin. Phys. Mech. Astron. 63, 231011 (2020).

[75] K. Agashe, Y. Cui, L. Necib, and J. Thaler, (In)direct detection of boosted dark matter, J. Cosmol. Astropart. Phys. 10 (2014) 062.

[76] J. Berger, Y. Cui, and Y. Zhao, Detecting boosted dark matter from the sun with large volume neutrino detectors, J. Cosmol. Astropart. Phys. 02 (2015) 005.

[77] J. F. Cherry, M. T. Frandsen, and I. M. Shoemaker, Direct Detection Phenomenology in Models Where the Products of Dark Matter Annihilation Interact with Nuclei, Phys. Rev. Lett. 114, 231303 (2015). 